\title{
Self-rated impulsivity in healthy individuals, substance use disorder and ADHD: psychometric properties of the Swedish Barratt impulsiveness scale
}

Lotfi Khemiri", Christoffer Brynte, Maija Konstenius, Joar Guterstam, Ingvar Rosendahl, Johan Franck and Nitya Jayaram-Lindström

\begin{abstract}
Background: Impulsivity is associated with several psychiatric disorders, including substance use disorders (SUD) and attention deficit hyperactivity disorder (ADHD). A widely used questionnaire to assess impulsivity is the Barratt Impulsiveness Scale (BIS), and the aim of the current study was to evaluate the psychometric properties of the Swedish version of the BIS (swe-BIS).

Methods: The original BIS was translated to Swedish and back-translated by an authorized translator. The swe-BIS was administered to healthy controls $(n=113)$, patients with alcohol use disorder $(n=97)$, amphetamine use disorder $(n=37)$ and attention deficit hyperactive disorder (ADHD; $n=26)$. A subset of subjects $(n=62)$ completed the swe-BIS twice within 1 week. Psychometric evaluation of the swe-BIS included assessment of different indices of reliability (internal consistency, test-retest and agreement) and validity (response processess, divergent and convergent). Confirmatory factor analyses (CFA) were performed to assess several indices of model fit in five different models based on previously suggested subscales.

Results: Cronbach's alpha for all swe-BIS items in the full sample was 0.89 , ranging from $0.78-0.87$ within the different subgroups. The Pearson test-retest correlation for total score was $0.78(p<0.001)$, with greater test-retest correlations within compared to across different subscales. The Bland-Altman plot indicated high level of agreement between test and retest. The healthy individuals had lower swe-BIS score compared to the patients $(\mathrm{t}(267.3)=-8.6 ; p<0.001)$, and the swe-BIS total score was also significantly different between each of the four participant groups ( $p<0.01$ for all group comparisons). Furthermore, swe-BIS had greater correlations with impulsivity related scales compared to non-impulsivity related scales. The CFA analyses indicated that while no suggested model showed an optimal fit, the best model fit indices was found for the 3-factor model.

Conclusions: The swe-BIS was found to have good to excellent psychometric properties with respect to the assessed indices of reliability and validity, supporting use of the scale in clinical research in both healthy individuals and patients with SUD and ADHD.
\end{abstract}

\footnotetext{
* Correspondence: lotfi.khemiri@ki.se

Department of Clinical Neuroscience, Centre for Psychiatry Research,

Karolinska Institutet \& Stockholm Health Care Services, Norra Stationsgatan

69, floor 7, 11364 Stockholm, Sweden
}

(c) The Author(s). 2021 Open Access This article is licensed under a Creative Commons Attribution 4.0 International License, which permits use, sharing, adaptation, distribution and reproduction in any medium or format, as long as you give appropriate credit to the original author(s) and the source, provide a link to the Creative Commons licence, and indicate if changes were made. The images or other third party material in this article are included in the article's Creative Commons licence, unless indicated otherwise in a credit line to the material. If material is not included in the article's Creative Commons licence and your intended use is not permitted by statutory regulation or exceeds the permitted use, you will need to obtain permission directly from the copyright holder. To view a copy of this licence, visit http://creativecommons.org/licenses/by/4.0/ The Creative Commons Public Domain Dedication waiver (http://creativecommons.org/publicdomain/zero/1.0/) applies to the data made available in this article, unless otherwise stated in a credit line to the data. 
Keywords: BIS, Barratt impulsiveness scale, Impulsivity, Motor impulsivity, Non-planning impulsivity, Attentional impulsivity, Psychometrics, Substance use disorder, Alcohol, Amphetamine, ADHD

\section{Introduction}

Impulsivity is a heterogenous personality and behavioural construct [1], present in the general population as well as in several psychiatric disorders, including substance use disorders (SUD), personality disorders and attention deficit hyperactivity disorder (ADHD). An impulsive act is in essence characterized by haste, lack of premeditation and disregard of the future consequences of the action. Even though no formal definition exists, a widely held definition presented by Moeller and colleagues is that impulsivity is "a predisposition toward rapid unplanned reactions to internal or external stimuli without regard to the negative consequences of these reactions to the impulsive individual or to others" [2].

There are a wide number of different scales and tests used to measure the construct of impulsivity. A distinction is often made between trait and state impulsivity: State impulsivity varies across time, is influenced by short-term experimental conditions and can be assessed by different laboratory based neuropsychological tests. Such tests aim to capture an individual's propensity toward impulsive behaviours, e.g., inability to inhibit prepotent responses, ability to plan for the future and the valuing of delayed rewards [3]. Trait impulsivity, or impulsiveness, in contrast, refers to the overall degree of impulsive behaviour in an individual that is relatively constant across time. This trait is instead assessed by self-rating questionnaires, where the subject is asked to rate how impulsive they are in general across several different behaviours and life situations. Common instruments used to assess trait impulsivity are the Impulsivity-Venturesomeness-Empathy scale [4], the Urgency, Premeditation, Perseverance, Sensation Seeking, Positive Urgency, Impulsive Behavior Scale [5] and the Barratt Impulsiveness Scale (BIS [6];).

The BIS is one of the most widely used self-rating questionnaires for impulsiveness. As reviewed by Stanford and colleagues, the BIS was developed by Barratt who originally conceptualized impulsiveness as a multidimensional construct which was orthogonal to anxiety [7]. After a series of revisions this work resulted in the BIS-11, which is the most recent version of the scale where impulsiveness is treated as a multidimensional construct [6]. Based on principal components analysis of the BIS-11, the trait of impulsiveness was conceptualized by Patton, Stanford and Barratt as consisting of six firstorder factors (attention, cognitive instability, motor, perseverance, self-control, cognitive complexity) which in turn formed three second-order factors entitled motor, attentional and non-planning impulsiveness. However, this model has been questioned given that other psychometric studies of the BIS has found better fit for twofactor solutions [8], while other studies have found best fit for three factor solutions, but with different item loadings compared to the original study [9]. In a systematic review of the psychometric properties of the BIS-11, it was concluded that even though the BIS-11 is a useful clinical tool to differentiate different clinical populations with high levels of impulsivity, there are conflicting data regarding the dimensions/subscales of the scale [10].

The BIS has been extensively used in clinical research and practice during the last decades [7], including studies of clinical populations with affective disorders [11, 12], prison inmates [13], antisocial personality disorder [14] and ADHD [15]. Furthermore, BIS scores have also been found to be elevated in patients with different forms of SUD, including alcohol [16, 17], cocaine [18] and opioids [19]. The BIS has been translated to other languages, including German [20, 21], Spanish [22], Norwegian [23], Chinese [24], Italian [9], Portuguese [25], Dialectal Arabic [26] and Japanese [27]. Considering the wide interest for impulsivity in clinical psychiatric research, we decided to translate the scale into Swedish. Because of the conflicting results of earlier studies, we also wanted to explore its psychometric properties in different populations.

The aim of the current was thus to perform a psychometric evaluation of a Swedish version of the BIS-11 (swe-BIS), including analyses of reliability, validity and confirmatory factor analyses in patients with SUD, ADHD and healthy volunteers. The main hypothesis was that the patient populations would exhibit elevated levels of impulsiveness compared to the control group (evidence of validity based on response processes). In addition we hypothesized that the swe-BIS outcomes should correlate to a higher degree with other impulsivity scales, and that the scale would have good test-retest and internal consistency properties.

\section{Methods \\ Adaptation of the BIS}

The English version of the BIS-11 [6] was translated and back-translated from English to Swedish by an authorized bilingual translator. A meeting was held with clinicians and clinical researchers to examine the individual items, and minor adjustments regarding exact phrasing of the items were discussed and agreed upon through 
consensus within the group. See supplementary material Table S1 for the Swedish translation of all BIS items.

\section{Participants}

The swe-BIS was administered to four groups of participants. All individuals were included in different research projects and were administered the swe-BIS as part of the baseline assessment. The first group was patients with alcohol use disorder (AUD) that were administered the questionnaire while taking part in two separate clinical treatment studies [28, 29]. The second group was patients with amphetamine use disorder $(\mathrm{AMPH})$ who completed the questionnaire when participating in a pharmacological fMRI study [30]. The third group comprised subjects with ADHD who were taking part in a pharmacological laboratory experimental study (Brynte et al., in preparation). The final group was healthy controls (HC) with no SUD or any other psychiatric or somatic illnesses, who were recruited from the community, to serve as control group to the AUD patient population in a separate study of cognitive functioning [31]. For each of the aforementioned studies, ethics approval was obtained from the Regional Ethics Review Board in Stockholm, and conducted in accordance with Good Clinical Practice and the declaration of Helsinki.

\section{Inclusion and exclusion criteria}

The major inclusion and exclusion criteria have been described in detail in the original studies [28-31]. In brief, the main inclusion criteria for the patients were current DSM-IV diagnosis of SUD (AUD or AMPH) or ADHD, no severe psychiatric disorder (e.g., bipolar disorder or schizophrenia), no severe somatic illness and no current suicidal ideation. All patients were required to be sober and drug free (assessed by alcometer and urine dip test) on test day, except for the subgroup of AMPH patients who were allowed to have positive urine dip test of central stimulants on the test day. For the HC group, the inclusion and exclusion criteria were similar except that they could not have had any current diagnosis or history of SUD, no illicit drug use the past 12 months, be sober and leave negative urine dip test for drugs of abuse on the day of testing.

\section{Procedure}

All participants underwent a medical evaluation by an M.D., including physical and psychiatric assessment using the Structured Clinical Interview for DSM-IV [32] or the Swedish DSM-5 version of the Mini-International Neuropsychiatric Interview [33]. The ADHD patients already had an existing ADHD diagnosis and confirmed by assessment of patient chart data. Alcohol, nicotine and drug consumption was assessed by the Time Line Follow Back (TLFB) interview [34]. All participants performed a breathalyzer test and urine dip test on the day of testing, and completed several self-rating questionnaires as part of baseline assessment before study participation.

\section{Questionnaires \\ Barratt impulsiveness scale, Swedish version}

The Swedish version of the Barratt Impulsiveness Scale (swe-BIS) comprises all the original 30 items from the original English BIS-11 [6]. Each item is a statement about impulsivity related thoughts/behaviours in different situations, and the subject is asked to rate how often he or she experiences such thoughts or behaviours on a 4-point scale ( 1 = "Rarely/never", 2 = "Occasionally", 3 = "Often" and 4="Almost always/always"). The BIS has three subscales namely motor, attention and nonplanning impulsiveness. Examples of items from each of the subscales are "I make up my mind quickly" (Motor impulsiveness), "I often have extraneous thoughts when thinking "(Attentional impulsiveness) and "I say things without thinking "(Non-planning impulsiveness).

The swe-BIS was administered to a majority of participants in paper form, and the subjects were asked to complete the questionnaire using a pen. A minority of patients (ADHD group; $n=26$ ) completed the same questionnaire through an electronic case record form (CRF) software and used a mouse to enter the responses on the computer screen. A subsample of subjects $(n=$ 62) completed the swe-BIS using pen and paper at home approximately 1 week prior to the test session.

\section{Montgomery-Åsberg depression self-rating scale}

The Montgomery-Åsberg Depression Self-Rating Scale (MADRS-S [35];) is a self-rating instrument consisting of 9 items regarding depression symptoms during the last 3 days. The items are rated on a scale from 0 to 6 , so the total score ranges from 0 to 54 , with higher scores indicating more severe levels of depressive symptoms.

\section{Adult ADHD self-report scale}

The World Health Organization Adult ADHD Selfreport Scale (ASRS [36];) is a screening questionnaire for adult ADHD according to the DSM-IV criteria. It consists of 18 questions (e.g., "How often do you have problems remembering appointments or obligations?") which are rated on a scale from 0 (never) to 4 (very often), resulting in a total score and two subscales of hyperactivity and inattention.

\section{Obsessive compulsive drinking scale}

The Obsessive Compulsive Drinking Scale (OCDS) is a widely used scale assessing compulsions and obsessions related to alcohol craving and drinking [37]. It consists of 14 items (e.g., "How much of your time when you're 
not drinking is occupied by ideas, thoughts, impulses, or images related to drinking?") which are rated from 0 to 4 resulting in a total sum score as well as two subscales of obsessions and drinking control/consequences.

\section{Swedish universities scales of personality}

The Swedish Universities Scales of Personality (SSP [38]; ) is a personality test, originally based on the Karolinska Scales of Personality, consisting of 91 individual items. Each item is a statement which is rated on a four-pointscale from 'does not apply at all' to 'applies completely'. The items are subdivided into 13 subscales e.g., impulsiveness and social desirability.

\section{Statistical analysis}

Sociodemographic and clinical background variables are presented as mean \pm standard deviation and fractions for continuous and categorical outcomes, respectively. Internal consistency was assessed by calculating Cronbach's alpha for all BIS items and each subscale, both in the total patient sample and within each of the different patient groups. Test-retest reliability was investigated by calculating Pearsons correlation, for total score and subscale scores, between the two time points of administration. To visually assess agreement between test-retest scores, Bland-Altman plots were created. In these plots, the $\mathrm{X}$ axis shows the mean difference between test sessions and the $\mathrm{Y}$ axis shows the average score across both test sessions, and it is expected that a majority of the difference scores would fall within +-2 (i.e., 1.96) standard deviations of the mean difference [39].

Validity is a complex unitary construct for which there are different forms of evidence. Evidence of validity based on response processes (commonly referred to as construct validity) was evaluated by a series of statistical tests where group was the independent variable and mean score of the swe-BIS total score and subscales were the dependent variables. First, t-tests were used to compare healthy controls and all patients on the sweBIS outcomes. Second, a oneway-ANOVA with group (HC, AUD, AMPH, ADHD) as independent variable was performed. Planned paired comparisons using t-tests without adjustment for multiple comparisons were performed between each group category. Because of lack of homogeneity of variances across groups assessed by Levene's test, Welch t-test and ANOVA was used. Evidence of convergent validity was evaluated by calculating Pearsons correlation between swe-BIS and ASRS total score, ASRS hyperactive, ASRS inattention and the SSP subscale of impulsivity. Finally, evidence of divergent validity in contrast, was investigated by correlating the swe-BIS to MADRS-S, OCDS and the SSP subscale of social desirability.
In the Confirmatory Factor Analyses (CFA), Mardia's Multivariate Normality Test was used to test the assumption of multivariate normality in the data. Comparison in model fit to data between several models was done by following fit measures: Chi-square value, root mean squared error of approximation (RMSEA), standardized root mean squared residual (SRMR), comparative fit index (CFI), Tucker-Lewis index (TLI) and Akaike's information criterion (AIC). Five models of the swe-BIS were tested: a single-factor (total score) model, three versions of a 2-factor model and finally a 3-factor model, based on the three BIS subscales of attentional, motor and non-planning impulsiveness.

All statistical analyses were performed using $\mathrm{R}$ [40] and the following packages: psych [41], BlandAltmanLeh [42], MVN [43], lavaan [44], semTools [45] and plyr [46].

\section{Results \\ Study participants}

In total 273 participants consisting of $\mathrm{HC}(n=113)$, patients with AUD $(n=97)$, AMPH $(n=37)$ and ADHD $(n=26)$, completed the swe-BIS questionnaire and were thus included in the study. Table 1 presents the sociodemographic and clinical background variables in detail for all participants and for each subgroup.

\section{Internal consistency reliability}

For all the 30 swe-BIS items in the full study sample, the Cronbach's alpha was .89 [95\% CI .87-.91], with slightly lower coefficients for each of the subscales of attentional (.79 [.76-.83]), motor (.72 [.67-.77]) and non-planning impulsiveness (.77 [.72-.81]). Similar but reduced Cronbach's alpha coefficients were found for the $\mathrm{HC}$ and the different patient populations (Table 2A).

\section{Test-retest reliability}

Among the $\mathrm{HC}$, a total of 62 individuals filled in the swe-BIS questionnaires on two occasions, approximately 1 week between completion of the questionnaires. There was a statistically significant test-retest correlation for swe-BIS total score $(r=.78 ; p<.001)$, as well as for the subscales of attentional $(r=.63 ; p<.001)$, motor $(r=.54$; $p<.001)$ and non-planning $(r=.77 ; p<.001)$ impulsiveness. The test-retest correlations were greater within each subscale, compared to the correlations across different subscales (Table 2B).

The test-retest results are presented as correlation between first and second test score (Fig. 1A) and a BlandAltman graph plotting the mean swe-BIS score against difference between test sessions (Fig. 1B). These plots illustrate that there seems to be slightly greater differences between test sessions with higher test scores. Furthermore, in the Bland-Altman plot the mean 
Table 1 Sociodemographic and clinical background variables in the full sample, consisting of healthy controls and patients with amphetamine use disorder (AMPH), alcohol use disorder (AUD) and attention deficit hyperactive disorder (ADHD). Values are presented as mean (standard deviation) unless stated otherwise

\begin{tabular}{|c|c|c|c|c|c|}
\hline & Full sample $(n=273)$ & Healthy controls $(n=113)$ & AMPH $(n=37)$ & AUD $(n=97)$ & ADHD $(n=26)$ \\
\hline Age & $45.5(10.4)$ & $45.8(11.5)$ & $44.4(9.9)$ & $47.7(7.6)$ & $36.9(11.2)$ \\
\hline N (\%) females & $109(40.0 \%)$ & $47(41.6 \%)$ & $0(0 \%)$ & $45(46.4 \%)$ & $17(65.4 \%)$ \\
\hline School years ${ }^{a}$ & $13.3(3.3)$ & $16.2(2.5)$ & $10.8(3.2)$ & $13.8(2.6)$ & $12.7(2.1)$ \\
\hline Nicotine daily use & $40 \%$ & $11.5 \%$ & $86.5 \%$ & $60.0 \%$ & $26.9 \%$ \\
\hline OCDS total ${ }^{\mathrm{b}}$ & $15.5(11.8)$ & $2.1(2.5)$ & NA & $23.8(6.4)$ & NA \\
\hline$\%$ drinking days $^{c}$ & $45(34)$ & $13(12)$ & NA & $72(21)$ & NA \\
\hline$\%$ amphetamine use days & $9.9(28)$ & 0 & $73(35)$ & 0 & 0 \\
\hline MADRS-S ${ }^{d}$ & $6.5(6.6)$ & $3.0(3.3)$ & NA & $7.6(6.5)$ & $11.5(8.3)$ \\
\hline ASRS total ${ }^{e}$ & $25.8(14.1)$ & $18.2(9.0)$ & $41.5(11.4)$ & $24.3(12.5)$ & $39.2(10.6)$ \\
\hline ASRS impulsivity ${ }^{f}$ & $11.4(6.3)$ & $10.1(5.1)$ & NA & $10.4(6.4)$ & $19.0(5.3)$ \\
\hline ASRS inattention ${ }^{f}$ & $11.6(7.4)$ & $7.9(4.9)$ & NA & $13.9(7.1)$ & $20.2(7.2)$ \\
\hline SSP impulsivity ${ }^{g}$ & $16.3(3.2)$ & $15.9(2.8)$ & NA & $16.9(3.6)$ & NA \\
\hline SSP social desirability ${ }^{9}$ & $20.1(2.6)$ & $20.6(2.7)$ & NA & $19.4(2.4)$ & NA \\
\hline
\end{tabular}

OCDS - Obsessive Compulse Drinking Scale; BIS - Barratt Impulsiveness Scale; MADRS-S - Montgomery-Åsberg Depression Self Rating Scale; ASRS - Adult ADHD

Self-report Scale; SSP - Swedish universities Scale of Personality. NA - no data available

${ }^{a}$ School year data available from 139 participants ${ }^{\text {b }}$ OCDS data available from 149 participants

c Drinking data available from 178 participants

d MADRS-S data available from 188 participants

e ASRS total score data available from 201 participants

${ }^{f}$ ASRS subscales data available from 135 individuals

g SSP data available from 123 individuals

average difference was close to zero (-0.2), and only three individuals out of $62(4.8 \%)$ had data points that fell outside the $95 \%$ CI of the mean difference.

\section{Validity based on response processes}

The HC had lower swe-BIS scores compared to the patient sample as a whole, for the BIS total score (HC: 56.2(8.9); Patients: 68.3(14.3); $\mathrm{t}(267.3)=-8.6 ; p<.001)$ as well as the attentional (HC: 12.9(2.8); Patients: 16.9(4.6); $\mathrm{t}(265.2)=-9.1 ; p<.001)$, motor (HC: 20.7(4.1); Patients: $24.3(5.3) ; \mathrm{t}(269.5)=-6.4 ; p<.001)$ and nonplanning (HC: 22.7(4.5); Patients: 27.1(6.4); $\mathrm{t}(271.0)=-$ $6.6 ; p<.001)$ subscales.

The ANOVA analysis including all subgroups (HC, AUD, AMPH, ADHD) of the swe-BIS total score indicated a statistically significant effect of group

Table 2 Internal consistency assessed by Cronbach's alpha [95\% confidence intervals] (A) and test-retest Pearson correlations (B) for the Swedish version of the Barratt Impulsiveness Scale (swe-BIS) administered to healthy controls, patients with amphetamine use disorder (AMPH), alcohol use disorder (AUD) and attention deficit hyperactive disorder (ADHD)

\begin{tabular}{|c|c|c|c|c|c|}
\hline & \multicolumn{5}{|c|}{ A. Internal consistency (Cronbach's alpha) } \\
\hline & Full sample $(n=273)$ & $\begin{array}{l}\text { Healthy controls }(n= \\
\text { 113) }\end{array}$ & $\mathrm{AMPH}(n=37)$ & $\operatorname{A\cup D}(n=97)$ & $\begin{array}{l}\operatorname{ADHD}(n= \\
26)\end{array}$ \\
\hline Swe-BIS all items & $.89[.87-.91]$ & $.78[.72-.89]$ & $.87[.82-.93]$ & $.82[.77-.87]$ & $.87[.80-.94]$ \\
\hline Swe-BIS attention & $.79[.76-.83]$ & $.60[.49-.71]$ & $.70[.56-.85]$ & $.72[.64-.80]$ & $.63[.42-.85]$ \\
\hline Swe-BIS motor & $.72[.67-.77]$ & $.50[.36-.64]$ & $.68[.53-.83]$ & $.62[.51-.73]$ & $.71[.56-.87]$ \\
\hline \multirow[t]{3}{*}{ Swe-BIS non-planning } & $.77[.72-.81]$ & $.70[.62-.79]$ & $.71[.58-.85]$ & $.64[.54-.75]$ & $.81[.70-.92]$ \\
\hline & \multicolumn{5}{|c|}{ B. Test-retest Pearson correlations across subscales } \\
\hline & $\begin{array}{l}\text { Swe-BIS total score (re- } \\
\text { rest) }\end{array}$ & $\begin{array}{l}\text { Swe-BIS attention (re- } \\
\text { test) }\end{array}$ & $\begin{array}{l}\text { Swe-BIS motor (re- } \\
\text { test) }\end{array}$ & $\begin{array}{l}\text { Swe-BIS non-planning (re- } \\
\text { test) }\end{array}$ & \\
\hline Swe-BIS total score (test) & $.78 P<0.001$ & & & & \\
\hline Swe-BIS attention (test) & & $.63 P<.001$ & $.18 P=.16$ & $.36 P=.005$ & \\
\hline Swe-BIS motor (test) & & $.39 P=.002$ & $.54 P<.001$ & $.26 P=.04$ & \\
\hline $\begin{array}{l}\text { Swe-BIS non-planning } \\
\text { (test) }\end{array}$ & & $.48 P<.001$ & $.38 P=.003$ & $.77 P<.001$ & \\
\hline
\end{tabular}




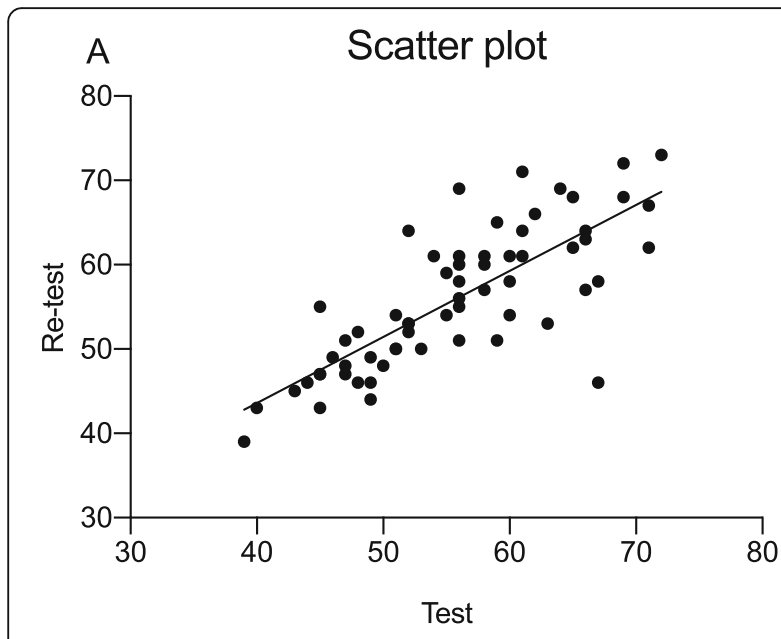

B Bland-Altman plot

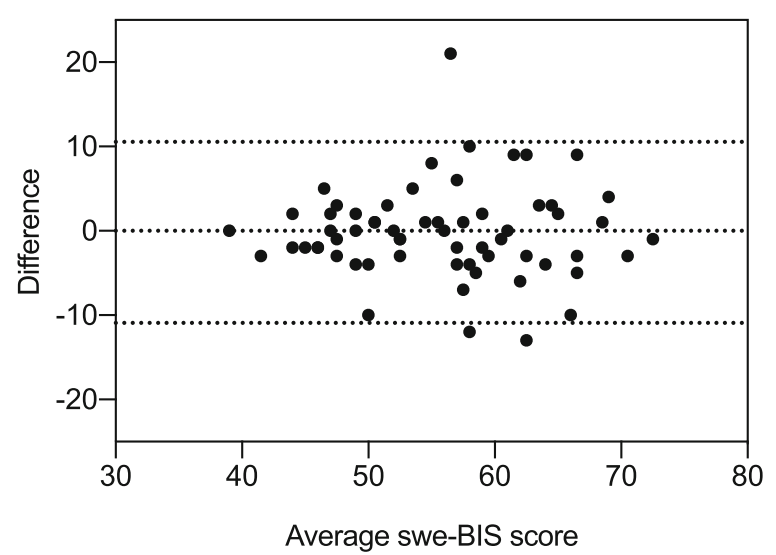

Fig. 1 The correlation between test and retest result on the Swedish version of the Barratt Impulsiveness Scale (swe-BIS) was 0.78 (A). The Bland-Altman plot presents the mean total swe-BIS score across both tests against the mean difference between test sessions (B). Three individuals (4.8\%) had mean difference scores outside the limits of +-2 standard deviations of the mean difference, indicating good agreement between test and re-test

$(\mathrm{F}(3,76.4)=49.6 ; p<.001 ; \mathrm{Fig} .2 \mathrm{~A})$. Post hoc comparisons found statistically significant differences for all comparisons between all four groups: The $\mathrm{HC}$ had significantly lower swe-BIS total score (HC mean: 56.2(8.9)) than patients with AMPH (Mean: 81.3; $p<.001$ ), AUD (Mean: $62.0 ; p<.001$ ) and ADHD (Mean: 73.5; $p<.001$ ). The AMPH group had highest score among the patients scoring significantly higher than both AUD $(p<.001)$ and ADHD $(p=.024)$, who in turn had higher scores than the AUD group $(p<.001)$. A similar pattern of results was found for all the swe-BIS subscales (Fig. 2B-D).

\section{Evidence of convergent and divergent validity}

The swe-BIS total score exhibited statistically significant correlations with the other impulsivity questionnaires i.e., ASRS $(r=.81 ; p<.001)$, ASRS impulsivity $(r=.67$; $p<.001)$, ASRS inattention $(r=.70 ; p<.001)$ and SSP impulsivity subscale $(r=.62 ; p<.001)$. Notably, the correlations were lower between swe-BIS total score and the non-impulsivity related scales i.e., MADRS-S $(r=.46$; $p<.001)$, OCDS $(r=.35 ; p<.001)$ and SSP social desirability $(r=-.20 ; p=.024)$.

\section{Confirmatory factor analysis}

The Mardia's Multivariate Normality Test was highly statistically significant for both skewness and kurtosis, signaling that data was not multivariate normal (z-kurtosis $=20.62, p<.001$ ). We therefore used the MLR (robust Maximum Likelihood) estimation method in all CFA of the swe-BIS scale. The results from the CFA of five different models of the swe-BIS scale are summarized in a number of common model fit indices in Table 3.

According to RMSEA, all the models have a mediocre fit (0.08-0.10) and none of them have an upper limit of the confidence interval below 0.08 which indicates a reasonable fit. No model showed a satisfactory value on CFI and TLI $(>0.90)$ or acceptable SRMR value $(<0.08)$, even though the 3-factor model had the best values among tested models. The AIC value was also lowest for the 3factor model. The best model for our data, based on an assessment of all the fit indices presented in Table 3 was thus the 3-factor model. Twenty-one of the thirty items had good factor loadings $(\geq 0.40)$ in the 3 -factor model. Of the nine items with a lower factor loading $(<0.40)$, only item $24(0.313)$ was from the attentional subscale, while item 15 (0.311), 27(0.353) and 29 (0.355) were from the non-planning subscale. Finally, in the motor subscale item 3 (0.275), 16 (0.253), 21 (0.268), 23 (0.301) and $30(0.335)$ had lower factor loading. All individual item factor loadings for the 3-factor model are presented in the supplementary material Table S2.

\section{Discussion}

In the current study, the swe-BIS was found to have overall satisfying psychometric properties when administered to both healthy individuals and clinical populations with AUD, AMPH and ADHD. Importantly, indices of both reliability and validity were in general good to excellent, suggesting that the scale can be administered to both healthy individuals as well as clinical patient populations with externalizing disorders.

The swe-BIS exhibited excellent internal consistency, as assessed by a Cronbach's alpha coefficient of .89 in the full sample, and .78-.87 in the different patient populations. There are different reports on cut-off values for the Cronbach's alpha, but often values in the range of $.70-.95$ are considered acceptable [47]. For the different subscales, the values of Cronbach's alpha were in general also acceptable with values of .72-.79 in the full sample. 


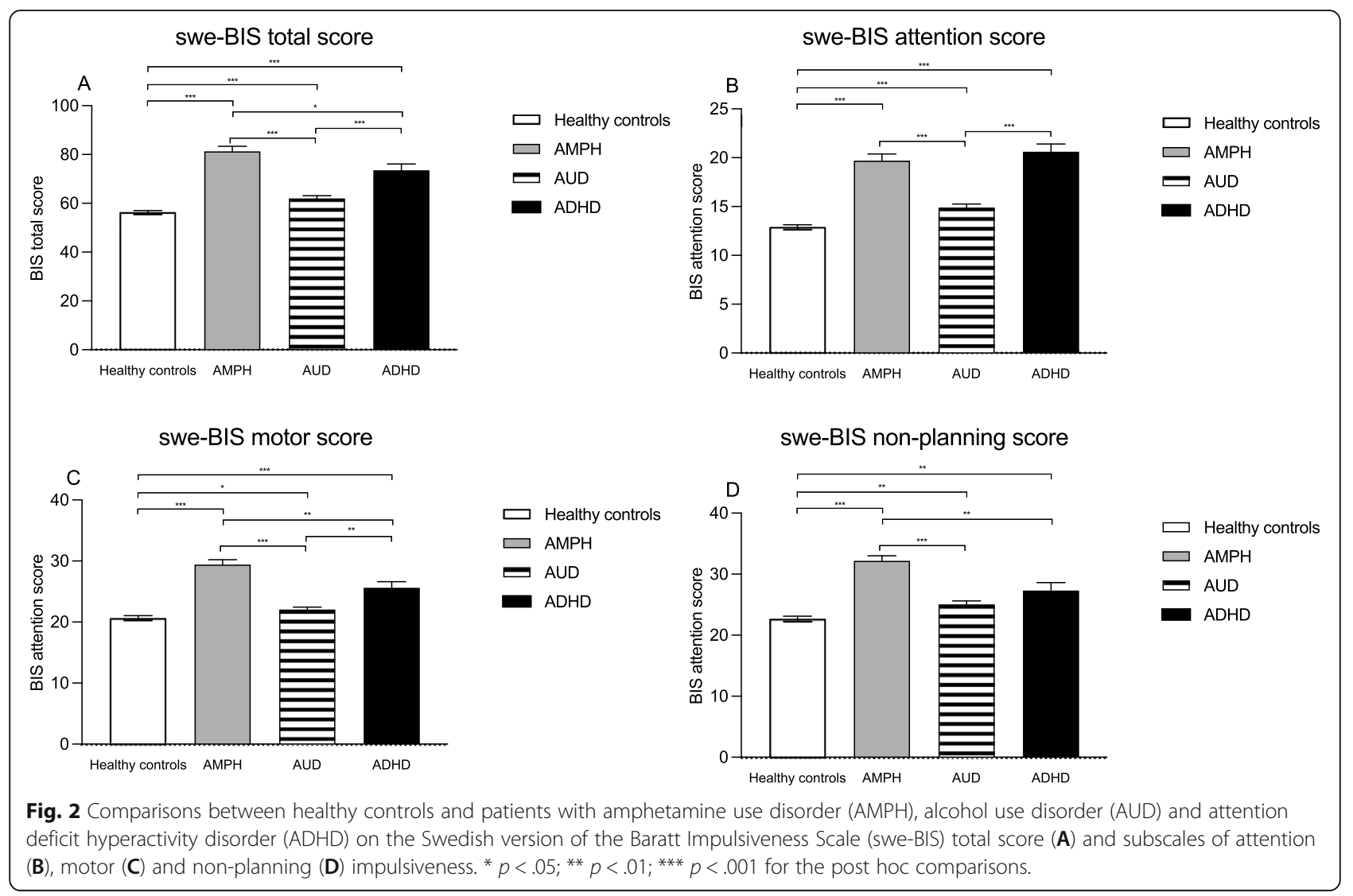

These results are similar to previously reported Cronbach's alpha values for several adaptations to different languages, as discussed in the review by Stanford and colleagues [7].

The correlation between test sessions was .78, and the test-retest correlations were greater within each subscale compared to across the subscales, indicating acceptable test-retest reliability for both the total score and subscales. Finally, the Bland-Altman plot illustrated that only a minority of participants had mean-difference scores outside the limits of +-2 standard deviations of the mean difference, indicating good agreement between test sessions. Importantly, since one of the completed BIS questionnaires was completed at home, these results also support that it is feasible to administer the scale outside of the clinic without supervision of research staff. Taken together, the Cronbach's alpha and testretest results indicate that the swe-BIS has good reliability, which is in line with conclusions from previous studies of the BIS-11 [10].

The validity analyses found that the swe-BIS was able to discriminate not only between $\mathrm{HC}$ and patients in general, but also was able to differentiate between different clinical patient populations. The findings of elevated

Table 3 Confirmatory Factor Analysis (CFA) results with model fit indices of five models of the Swedish version of the Barratt Impulsiveness Scale (swe-BIS)

\begin{tabular}{|c|c|c|c|c|c|c|c|}
\hline Model & $x^{2}$ & Df & RMSEA [90\% Cl] & SRMR & $\mathrm{CFI}$ & TLI & AIC \\
\hline 1-factor model & 1166.436 & 405 & $0.083[0.078-0.088]$ & 0.084 & 0.691 & 0.668 & $19,102.624$ \\
\hline 2-factor model, 1st version ${ }^{\mathrm{a}}$ & 1137.630 & 404 & $0.082[0.076-0.087]$ & 0.083 & 0.702 & 0.679 & $19,071.382$ \\
\hline 2-factor model, 2nd version ${ }^{\mathrm{b}}$ & 1130.500 & 404 & $0.081[0.076-0.086]$ & 0.084 & 0.705 & 0.682 & $19,061.207$ \\
\hline 2-factor model, 3rd version ${ }^{c}$ & 1152.555 & 404 & $0.082[0.077-0.088]$ & 0.084 & 0.696 & 0.673 & $19,087.222$ \\
\hline 3-factor model ${ }^{d}$ & 1111.630 & 402 & $0.080[0.075-0.086]$ & 0.084 & 0.712 & 0.688 & $19,042.103$ \\
\hline
\end{tabular}

$X^{2}$ chi square value, RMSEA Root mean squared error of approximation, SRMR Standardized root mean squared residual, CFI Comparative fit index, TLI Tucker-Lewis index, AIC Akaike's information criterion

${ }^{a}$ Attentional - Motor/Nonplanning

${ }^{\mathrm{b}}$ Motor - Attentional/Nonplanning

cNonplanning - Attentional/Motor

${ }^{\mathrm{d}}$ The model with best fit 
self-rated impulsiveness in SUD and ADHD are in line with several previous studies [15-19]. Notably, the BIS scores for the AUD group in the current study was significantly lower than the AMPH patients. This is likely in part explained by the fact that the different patient populations had different severity of dependence. While the AUD patients in general had a stable social situation and a wider distribution of number of DSM-IV alcohol dependence criteria fulfilled (range 3-7; Mean: 5.0 (1.2)), the AMPH group had more social problems and consistently severe levels of substance dependence (range 5-7; Mean: 6.7 (0.7)). These results are in line with previous studies that have found higher BIS scores in early onset AUD thought to reflect a more severe form of the disorder [48]. Finally, we found that the swe-BIS score correlated to a higher degree with other indices of impulsivity, and to a lesser degree with clinical selfrating scales measuring depressive symptoms, craving or other personality traits. Taken together the swe-BIS exhibited satisfying validity and our results suggest that it can identify group differences regarding impulsiveness within and between different externalizing disorders such as SUD and ADHD. Furthermore, our results from the convergent/divergent validity analyses suggest that the swe-BIS captures specific aspects of impulsiveness, and not a general propensity of responding high scores on questionnaires in general.

The confirmatory factor analysis indicated that none of the models achieved a good fit to the observed data, based on different fit indices such as CFI, TLI or SRMR. Of all the tested models however, the 3-factor model had the lowest AIC value, suggesting that even though far from perfect the 3-factor model indeed provided the best fit to the observed data. However, it is important to note that 9 items had a poor factor loading $(<0.4)$ and these items related to all three factors, but mainly to motor (five items) and non-planning (three items) subscales. In future revisions of the swe-BIS these items may be considered to revise or retranslate in order to improve the factor structure of the scale. It is important however, that almost all studies of different BIS versions have found different results for the optimal factor structure of the BIS scale [10], likely reflecting cultural differences in interpretation of certain items and the overall heterogeneity of the impulsiveness construct.

While the current study has several strengths, such as inclusion of different patient populations and detailed assessment (and exclusion) of severe psychiatric comorbidity, there are some important limitations in need of discussion. First of all, sample size was limited especially for the patient groups of AMPH and ADHD, so the estimates in these samples are less certain than for the HC and AUD groups. Second, test-retest data was only available for a subsample of the HC $(n=62)$. The results regarding test-retest are therefore not necessarily generalizable to the clinical patient populations. Furthermore, the timing of the test-retest was on average 1 week, so no conclusions regarding long-term test-retest and agreement can be made based on this study. Finally, data for the convergent and divergent validity analyses were only available in a subsample of $\mathrm{HC}$ and $\mathrm{AD}$ patients $(n=123)$, so therefore we cannot infer that the same results hold for AMPH or ADHD.

\section{Conclusions}

In summary, the swe-BIS exhibited good to excellent psychometric properties regarding reliability and validity and can be utilized in healthy individuals and patients with externalizing disorders such as SUD and ADHD. While far from optimal, the 3-factor solution provided the best model fit suggesting that the three subscales of motor, non-planning and attentional impulsiveness can be analyzed albeit with caution. In order to investigate the general validity of the swe-BIS further, future studies could include patients with other psychiatric disorders (e.g., affective disorders or personality disorders) or neurological disorders (e.g., Parkinson's disease) where the disorder or treatment response might be characterized by impulsive behavior.

\section{Abbreviations \\ ADHD: Attention Deficit Hyperactivity Disorder; AIC: Akaike's Information Criterion; AMPH: Amphetamine Use Disorder; ASRS: The World Health Organization Adult ADHD Self-report Scale; AUD: Alcohol Use Disorder; BIS: Barratt Impulsiveness Scale; CFA: Confirmatory Factor Analyses; CFI: Comparative Fit Index; HC: Healthy Controls; MADRS-S: Montgomery- Åsberg Depression Self-Rating Scale; MLR: Robust Maximum Likelihood; OCDS: The Obsessive Compulsive Drinking Scale; RMSEA: Root Mean Squared Error of Approximation; SRMR: Standardized Root Mean Squared Residual; SSP: The Swedish Universities Scales of Personality; SUD: Substance Use Disorder; Swe-BIS: Swedish version of the Barratt Impulsiveness Scale; TLFB: Time Line Follow Back; TLI: Tucker-Lewis Index}

\section{Supplementary Information}

The online version contains supplementary material available at https://doi. org/10.1186/s12888-021-03462-1.

Additional file 1: Table S1. The translated version of the Swedish version of the Barratt Impulsiveness Scale (swe-BIS). Table S2. Individual item factor loadings for the best fitting 3-factor model for the Swedish version of the Barratt Impulsiveness Scale (swe-BIS).

\section{Acknowledgements}

We thank research staff Margareta Gard-Hedander, Else-Britt Hillner, Rebecka Broman, Daniella Souma, Angela Stünkel, Amanda Bäcker, Maria Östman, Zakarias Thorin and Camilla Hellspong for assistance in data collection.

\section{Authors' contributions}

LK, CB and JG collected the data. LK and IR performed the statistical analysis. $L K, C B, M K, J G, I R$, JF and NJL contributed to the study design, interpretation of results and writing of the manuscript. All authors read and approved the final manuscript. 


\section{Funding}

The study was supported by grants provided to NJL by the Stockholm County Council (20170512), Stiftelsen Söderström Königska Sjukhemmet (SLS-750801, SLS-780001, SLS-313731), Swedish Society of Medicine (SLS500921 and SLS573691) and the Research Council of the Swedish Alcohol Retailing Monopoly (FO2012-0043). LK, JG and CB were supported by the Stockholm County Council combined clinical residency and PhD training program (project grants 20170264, 20110111, K0141-2015). LK received grants from the Professor Bror Gadelius Memorial Fund and Psykiatrifonden. JF received grants from the Research Council for Working Life and Health (2013-1849) and the Swedish Research Council (2012-02607). The funding bodies had no direct role in study design, data collection, analysis/interpretation or writing of the manuscript. Open Access funding provided by Karolinska Institute.

\section{Availability of data and materials}

The datasets generated and/or analysed during the current study are not publicly available due to individual privacy but are available in summary/ group level form from the corresponding author on reasonable request.

\section{Declarations}

\section{Ethics approval and consent to participate}

The data presented in the current manuscript was collected as part of other studies, which all have been approved by the Regional Ethics Review Board in Stockholm prior to study initiation, and conducted in accordance with Good Clinical Practice and the declaration of Helsinki. All participants were given oral and written information regarding the study procedures, and provided informed consent to participate in the study.

\section{Consent for publication}

All participants provided informed consent, including consent to publish scientific manuscripts based on collected data, in order to participate in the study.

\section{Competing interests}

The authors declare that they have no competing interests.

\section{Received: 20 January 2021 Accepted: 27 August 2021}

\section{Published online: 18 September 2021}

\section{References}

1. Evenden JL. Varieties of impulsivity. Psychopharmacology. 1999;146(4):34861. https://doi.org/10.1007/PL00005481.

2. Moeller FG, Barratt ES, Dougherty DM, Schmitz JM, Swann AC. Psychiatric aspects of impulsivity. Am J Psychiatry. 2001;158(11):1783-93. https://doi. org/10.1176/appi.ajp.158.11.1783.

3. Verdejo-García A, Lawrence AJ, Clark L. Impulsivity as a vulnerability marker for substance-use disorders: review of findings from high-risk research, problem gamblers and genetic association studies. Neurosci Biobehav Rev. 2008;32(4):777-810. https://doi.org/10.1016/j.neubiorev.2007.11.003.

4. Eysenck SBG, Pearson PR, Easting G, Allsopp JF. Age norms for impulsiveness, venturesomeness and empathy in adults. Personal Individ Differ. 1985;6(5):613-9. https://doi.org/10.1016/0191-8869(85)90011-X.

5. Whiteside SP, Lynam DR. Understanding the role of impulsivity and externalizing psychopathology in alcohol abuse: application of the UPPS impulsive behavior scale. Exp Clin Psychopharmacol. 2003;11(3):210-7. https://doi.org/10.1037/1064-1297.11.3.210.

6. Patton JH, Stanford MS, Barratt ES. Factor structure of the Barratt impulsiveness scale. J Clin Psychol. 1995;51(6):768-74. https://doi.org/10.1 002/1097-4679(199511)51:6<768::AID-JCLP2270510607>3.0.CO;2-1.

7. Stanford MS, Mathias CW, Dougherty DM, Lake SL, Anderson NE, Patton JH. Fifty years of the Barratt impulsiveness scale: an update and review. Personal Individ Differ. 2009;47(5):385-95. https://doi.org/10.1016/j.paid.2009. 04.008.

8. Reise SP, Moore TM, Sabb FW, Brown AK, London ED. The Barratt impulsiveness Scale-11: reassessment of its structure in a community sample. Psychol Assess. 2013;25(2):631-42. https://doi.org/10.1037/a0032161.

9. Fossati A, Di Ceglie A, Acquarini E, Barratt ES. Psychometric properties of an Italian version of the Barratt impulsiveness Scale-11 (BIS-11) in nonclinical subjects. J Clin Psychol. 2001;57(6):815-28. https://doi.org/10.1002/jclp.1051.
10. Vasconcelos AG, Malloy-Diniz L, Correa H. Systematic review of psychometric properties of Barratt Impulsiveness Scale Version 11 (BIS-11) Clinical Neuropsychiatry: Journal of Treatment Evaluation. 2012:61-74.

11. Swann AC, Steinberg JL, Lijfiijt M, Moeller FG. Impulsivity: differential relationship to depression and mania in bipolar disorder. J Affect Disord 2008:106(3):241-8. https://doi.org/10.1016/j.jad.2007.07.011.

12. MAM P, Hatch JP, Glahn DC, Monkul ES, Sanches M, Najt $P$, et al. Trait impulsivity in patients with mood disorders. J Affect Disord. 2007;100:227-31.

13. Smith $P$, Waterman $M$, Ward N. Driving aggression in forensic and nonforensic populations: relationships to self-reported levels of aggression, anger and impulsivity. Br J Psychol. 2006;97(Pt 3):387-403. https://doi.org/1 0.1348/000712605X79111.

14. Swann AC, Lijffijt M, Lane SD, Steinberg JL, Moeller FG. Trait impulsivity and response inhibition in antisocial personality disorder. J Psychiatr Res. 2009: 43(12):1057-63. https://doi.org/10.1016/j.jpsychires.2009.03.003.

15. Roberts W, Milich R, Fillmore MT. The effects of Preresponse cues on inhibitory control and response time in adults with ADHD. J Atten Disord. 2016;20(4):317-24. https://doi.org/10.1177/1087054713495737.

16. Bjork JM, Hommer DW, Grant SJ, Danube C. Impulsivity in abstinent alcoholdependent patients: relation to control subjects and type 1-/type 2-like traits. Alcohol. 2004;34(2-3):133-50. https://doi.org/10.1016/j.alcohol.2004.06.012.

17. Ketzenberger KE, Forrest L. Impulsiveness and compulsiveness in alcoholics and nonalcoholics. Addict Behav. 2000;25(5):791-5. https://doi.org/10.1016/ S0306-4603(00)00062-9.

18. Moeller FG, Barratt ES, Fischer CJ, Dougherty DM, Reilly EL, Mathias CW, et al. P300 event-related potential amplitude and impulsivity in cocainedependent subjects. Neuropsychobiology. 2004;50(2):167-73. https://doi. org/10.1159/000079110

19. Peters L, Soyka M. Interrelationship of opioid dependence, impaired impulse control, and depressive symptoms: an open-label cross-sectional study of patients in maintenance therapy. Neuropsychobiology. 2019;77(2):73-82. https://doi.org/10.1159/000494697.

20. Preuss UW, Rujescu D, Giegling I, Watzke S, Koller G, Zetzsche T, et al Psychometric evaluation of the German version of the Barratt impulsiveness scale. Nervenarzt. 2008;79(3):305-19. https://doi.org/10.1 007/s00115-007-2360-7.

21. Hartmann AS, Rief W, Hilbert A. Psychometric properties of the German version of the Barratt impulsiveness scale, version 11 (Bis-11) for adolescents. Percept Mot Skills. 2011;112(2):353-68. https://doi.org/10.2466/ 08.09.10.PMS.112.2.353-368.

22. Martínez-Loredo V, Fernández-Hermida JR, Fernández-Artamendi S, Carballo U, García-Rodríguez O. Spanish adaptation and validation of the Barratt impulsiveness scale for early adolescents (BIS-11-a). Int J Clin Health Psychol. 2015;15(3):274-82. https://doi.org/10.1016/j.ijchp.2015.07.002.

23. Lindstrøm JC, Wyller NG, Halvorsen MM, Hartberg S, Lundqvist C. Psychometric properties of a Norwegian adaption of the Barratt impulsiveness Scale-11 in a sample of Parkinson patients, headache patients, and controls. Brain Behav. 2017;7(1):e00605. https://doi.org/10.1 002/brb3.605.

24. Yao S, Yang H, Zhu X, Auerbach RP, Abela JRZ, Pulleyblank RW, et al. An examination of the psychometric properties of the Chinese version of the Barratt impulsiveness scale, 11th version in a sample of Chinese adolescents. Percept Mot Skills. 2007;104(3 Pt 2):1169-82. https://doi.org/10.2466/pms.1 04.4.1169-1182.

25. von Diemen L, Szobot CM, Kessler F, Pechansky F. Adaptation and construct validation of the Barratt impulsiveness scale (BIS 11) to Brazilian Portuguese for use in adolescents. Braz J Psychiatry. 2007;29(2):153-6. https://doi.org/1 0.1590/S1516-44462006005000020.

26. Ellouze F, Ghaffari O, Zouari O, Zouari B, M'rad MF. Validation of the dialectal Arabic version of Barratt's impulsivity scale, the BIS-11. Encephale. 2013;39(1): 13-8. https://doi.org/10.1016/j.encep.2012.06.034.

27. Someya T, Sakado K, Seki T, Kojima M, Reist C, Tang SW, et al. The Japanese version of the Barratt impulsiveness scale, 11th version (BIS-11): its reliability and validity. Psychiatry Clin Neurosci. 2001;55(2):111-4. https://doi.org/10.1 046/j.1440-1819.2001.00796.x

28. Khemiri L, Steensland P, Guterstam J, Beck O, Carlsson A, Franck J, et al. The effects of the monoamine stabilizer (-)-OSU6162 on craving in alcohol dependent individuals: a human laboratory study. Eur Neuropsychopharmacol. 2015;25(12):2240-51. https://doi.org/10.1016/j. euroneuro.2015.09.018. 
29. Khemiri L, Brynte C, Stunkel A, Klingberg T, Jayaram-Lindström N. Working memory training in alcohol use disorder: a randomized controlled trial. Alcohol Clin Exp Res. 2019;43(1):135-46. https://doi.org/1 $0.1111 /$ acer. 13910.

30. Guterstam J, Jayaram-Lindström N, Berrebi J, Petrovic P, Ingvar M, Fransson $P$, et al. Cue reactivity and opioid blockade in amphetamine dependence: a randomized, controlled fMRI study. Drug Alcohol Depend. 2018;191:91-7. https://doi.org/10.1016/j.drugalcdep.2018.06.023.

31. Khemiri L, Franck J, Jayaram-Lindström N. Effect of alcohol use disorder family history on cognitive function. Psychol Med. 2020:1-13. https://doi. org/10.1017/S003329172000238X. Epub ahead of print. PMID: 32662376.

32. American Psychiatric Association. Diagnostic and statistical manual of mental disorders (4th ed., text rev.). Washington DC; 2000.

33. Sheehan DV, Lecrubier $Y$, Sheehan KH, Amorim P, Janavs J, Weiller E, et al. The Mini-International Neuropsychiatric Interview (M.I.N.I.): the development and validation of a structured diagnostic psychiatric interview for DSM-IV and ICD-10. J Clin Psychiatry. 1998;59(Suppl 20):22-33 quiz 34-57.

34. Sobell L, Sobell M. Timeline follow-back: a technique for assessing selfreported ethanol consumption. In: Litten R, Allen J, editors. Measuring alcohol consumption: psychosocial and biological methods. Totowa: Humana Press; 1992. p. 41-72. https://doi.org/10.1007/978-1-4612-0357-5_3.

35. Svanborg P, Asberg M. A comparison between the Beck depression inventory (BDI) and the self-rating version of the Montgomery Asberg depression rating scale (MADRS). J Affect Disord. 2001;64(2-3):203-16. https://doi.org/10.1016/50165-0327(00)00242-1.

36. Kessler RC, Adler L, Ames M, Demler O, Faraone S, Hiripi E, et al. The World Health Organization adult ADHD self-report scale (ASRS): a short screening scale for use in the general population. Psychol Med. 2005;35(2):245-56. https://doi.org/10.1017/S0033291704002892.

37. Anton RF, Moak DH, Latham P. The obsessive compulsive drinking scale: a self-rated instrument for the quantification of thoughts about alcohol and drinking behavior. Alcohol Clin Exp Res. 1995;19(1):92-9. https://doi.org/1 0.1111/j.1530-0277.1995.tb01475.x.

38. Gustavsson JP, Bergman H, Edman G, Ekselius L, von Knorring L, Linder J. Swedish universities scales of personality (SSP): construction, internal consistency and normative data. Acta Psychiatr Scand. 2000;102(3):217-25. https://doi.org/10.1034/j.1600-0447.2000.102003217.x.

39. Bland JM, Altman DG. Statistical methods for assessing agreement between two methods of clinical measurement. Lancet. 1986; (8476):307-10.

40. R Core Team. R: a language and environment for statistical computing. Vienna: R Foundation for Statistical Computing; 2017.

41. Revelle W. psych: Procedures for Psychological, Psychometric, and Personality Research. 2020. https://CRAN.R-project.org/package=psych.

42. Lehner B. BlandAltmanLeh. 2015. https://cran.r-project.org/web/packages/ BlandAltmanLeh/index.html.

43. Korkmaz S, Goksuluk D, Zararsiz G. MVN: an R package for assessing multivariate normality. R Journal. 2014;6(2):151-62. https://doi.org/10.32614/ RJ-2014-031.

44. Rosseel Y. Lavaan: an R package for structural equation modeling. I Stat Softw. 2012:48(2):1-36. https://doi.org/10.18637/jss.v048.i02

45. Jorgensen TD, Pornprasertmanit S, Schoemann AM, Rosseel Y. semTools: Useful tools for structural equation modeling. R package version $0.5-3$. https://CRAN.R-project.org/package=semTools.

46. Wickham $\mathrm{H}$. The Split-apply-combine strategy for data analysis. J Stat Softw. 2011;40(1):1-29. https://doi.org/10.18637/jss.v040.i01

47. Tavakol M, Dennick R. Making sense of Cronbach's alpha. Int J Med Educ. 2011;2:53-5. https://doi.org/10.5116/ijme.4dfb.8dfd.

48. Dom G, Hulstijn W, Sabbe B. Differences in impulsivity and sensation seeking between early- and late-onset alcoholics. Addict Behav. 2006;31(2): 298-308. https://doi.org/10.1016/j.addbeh.2005.05.009.

\section{Publisher's Note}

Springer Nature remains neutral with regard to jurisdictional claims in published maps and institutional affiliations.

Ready to submit your research? Choose BMC and benefit from:

- fast, convenient online submission

- thorough peer review by experienced researchers in your field

- rapid publication on acceptance

- support for research data, including large and complex data types

- gold Open Access which fosters wider collaboration and increased citations

- maximum visibility for your research: over $100 \mathrm{M}$ website views per year

At $\mathrm{BMC}$, research is always in progress.

Learn more biomedcentral.com/submissions 